\title{
Generalizations of Matérn's hard-core point processes
}

\author{
J. Teichmann ${ }^{a}$, F. Ballani ${ }^{a}$, K. G. van den Boogaart ${ }^{b}$
}

September 13, 2012

\author{
${ }^{a}$ Institut für Stochastik, Technische Universität Bergakademie Freiberg, \\ Prüferstr. 9, D-09596 Freiberg, Germany, \\ jakob.teichmann@math.tu-freiberg.de / ballani@math.tu-freiberg.de \\ ${ }^{b}$ Helmholtz Institute Freiberg for Resource Technology, TU Bergakademie Freiberg, \\ Halsbrücker Str. 34, D-09599 Freiberg, Germany, \\ boogaart@math.tu-freiberg.de
}

\begin{abstract}
Matérn's hard-core processes are valuable point process models in spatial statistics. In order to extend their field of application, Matérn's original models are generalized here, both as point processes and particle processes. The thinning rule uses a distance-dependent probability function, which controls deletion of points close together. For this general setting, explicit formulas for first- and second-order characteristics can be given. Two examples from materials science illustrate the application of the models.
\end{abstract}

Key words: Point process, marked Poisson process, Matérn hard-core process, dependent thinning, independent thinning, pair correlation function.

\section{Introduction}

The present paper aims to generalize Matérn's well-known first and second hard-core point processes. These point process models, introduced by B. Matérn Matérn, 1960 , 1986, are typical examples for models derived from Poisson point processes, the latter being an important basis for constructing more complicated point processes, random sets and fibre processes at all [Stoyan et al. 1995]. They have been successfully applied to real data, for instance, they have been used in ecological [e. g. Picard et al. 2005, Stoyan, 1987, Warren, 1972 and CSMA network modeling [e.g. Baccelli and Błaszczyszyn, 2009, Busson and Chelius, 2009, Haenggi, 2011] as well as geographical analysis |Stoyan, 1988.

Matérn's first and second hard-core point processes are derived by applying a specific thinning rule to a homogeneous Poisson point process in $\mathbb{R}^{d}$. As such they are 
important examples for dependent thinning [Illian et al., 2008, Stoyan, 1988] where the thinning depends on the underlying process, somehow. For instance, the Matérn $I$ hard-core point process is obtained by deleting every point in the process with its nearest neighbor closer than a given hard-core distance [cf. Matérn, 1960, 1986]. In general, a thinning operation or rule [Illian et al. 2008 determines which points in the basic process are deleted. For example, such thinnings drive the evolution of plant communities due to competition-induced mortality [Batista and Maguire, 1998].

In contrast to depend thinning, the well-known $p$ - and $p(x)$-thinning approaches described in [Illian et al. 2008, Section 6.2.1] or Daley and Vere-Jones, 2008, Section 11.3] use independent thinning. That means that the thinning operation is independent of the configuration of the underlying point process and, at position $x$, a point will be deleted with some deterministic probability $1-p$ or $1-p(x)$, respectively.

In the following, the way of thinning a Poisson point process in order to obtain a Matérn hard-core point process is generalized in two directions. The first idea is to combine both independent and dependent thinning which can simply be interpreted as a subsequent independent thinning of a dependently thinned point process. The second idea is that in the dependent thinning a distance-dependent probability function $f$ controls deletion of points which are close together. This means that, depending on the distance to its neighbors, a point will be deleted only with some probability and not surely as is the case, e.g., in the Matérn I thinning rule. Thus it seems to be justified to speak of a probabilistic thinning rule in what follows.

An application of appropriate probabilistic thinning rules to Poisson point processes then leads to a class of point processes which are generalizations of the Matérn hardcore point processes. As distinguished from Gibbs point processes [Stoyan et al., 1995, Illian et al., 2008, Daley and Vere-Jones, 2008], explicit formulas for first- and secondorder characteristics can then still be derived. By means of several examples for the probability function $f$ it is shown that soft-core, hard-core as well as aggregative point processes can be obtained by this approach which reveals its high flexibility.

This paper is organized as follows. In Section 2 first the generalization of the Matérn I hard-core point process based on the thinning of a homogeneous Poisson process is introduced and first-order and second-order characteristics are given. Based on the idea of Stoyan and Stoyan 1985 and Månsson and Rudemo [2002 to extend the original Matérn II hard-core model by giving each point a random radius, where in Månsson and Rudemo, 2002 as a special case also a respective extension of the Matérn I process is covered, in Section 3 the dependent thinning model is further enhanced to marked point processes which is useful to model particle systems. Section 4 contains then a discussion of a related generalization of Matérn-II-type point processes. Finally, in Section 5, the applicability of the new models is illustrated by means of two data sets. 


\section{Probabilistic thinning model - Matérn I case}

\subsection{Model description}

Let $\Phi$ be a homogeneous Poisson point process in $\mathbb{R}^{d}$ with intensity $\lambda>0$ [see Daley and Vere-Jones, 1988 on a probability space $\left.\left.(\Omega, \mathcal{F}, \mathbb{P}), p_{0} \in\right] 0,1\right]$, and $f:[0, \infty[\longrightarrow$ $[0,1]$ be a measurable function. Denote by $\|\cdot\|$ Euclidean distance in $\mathbb{R}^{d}$. From $\Phi$ a new model $\Phi_{\text {th }}$ is derived by applying the following probabilistic dependent thinning rule to $\Phi$. A point $x \in \Phi$ is retained with probability

$$
p(x, \Phi)=p_{0} \prod_{\substack{y \in \Phi \\ y \neq x}}[1-f(\|x-y\|)]
$$

independently from deleting or retaining other points of $\Phi$. This means that two points a distance $r>0$ apart delete each other independently with probability $f(r)$. Independently from deleting due to pairwise interaction, each (surviving) point is (then) deleted with probability $1-p_{0}$.

Since the homogeneous Poisson process $\Phi$ is both stationary and isotropic and the thinning rule is independent both from location and direction, the thinned point process $\Phi_{\text {th }}$ is stationary and isotropic as well.

In the following we will sometimes write $\operatorname{MatI}\left[\lambda, p_{0}, f\right]$ for the distribution of $\Phi_{\text {th }}$.

Remark 1. Taking $p_{0}=1$ and $f=\mathbb{1}_{[0, R]}$ for some $R>0$, the point process $\Phi_{\text {th }}$ coincides with the Matérn I hard-core process with hard-core distance $R$ since then any two points a distance $r \leq R$ apart delete each other with probability 1, i. e. almost surely.

Remark 2. The thinning rule (1) could be refined by making the retention probability dependent also on three-point or further multi-point configurations, or, more general, on any functional which depends both on the point $x \in \Phi$ and the point pattern $\Phi$. An example for the latter would be the number $n_{R}(x, \Phi)$ of neighbors of $x \in \Phi$ within a certain distance $R>0$ which is, e.g., directly used in the definition of Strauss point processes [Strauss, 1975]. In fact, even the choice $f=f_{0} \mathbb{1}_{[0, R]}$ for some $\left.f_{0} \in\right] 0,1[$ results in a retention probability

$$
p(x, \Phi)=p_{0}\left(1-f_{0}\right)^{n_{R}(x, \Phi)} .
$$

\subsection{First- and second-order characteristics}

As is known for the original Matérn I hard-core point process, explicit formulas both for its intensity and its pair correlation function [Daley and Vere-Jones, 1988, Illian et al. 2008, Møller and Waagepetersen, 2003, Stoyan et al. |1995. can be stated |Daley and Vere-Jones, 1988, Illian et al., 2008, Matérn, 1960, 1986, Stoyan and Stoyan, 1985]. Although the definition of the thinned point process $\Phi_{\text {th }}$ is more complicated, arguments similar to that given for instance in Daley and Vere-Jones, 1988] can be used to derive the subsequent expressions in Theorems 3 and 4 for both the intensity 
and the pair correlation function of $\Phi_{\mathrm{th}}$. The respective proofs are omitted here due to the fact that the model MatI $\left[\lambda, p_{0}, f\right]$ also appears as a special case of another model introduced later in Section 3 .

Theorem 3 (Intensity). The intensity $\lambda_{\mathrm{th}}$ of the thinned point process $\Phi_{\mathrm{th}}$ is

$$
\lambda_{\mathrm{th}}=\lambda p_{0} \exp \left(-\lambda d b_{d} \int_{0}^{\infty} f(r) r^{d-1} \mathrm{~d} r\right),
$$

where $b_{d}$ denotes the volume of the unit ball in $\mathbb{R}^{d}$.

In case the integral in Theorem 3 is infinite, the resulting intensity $\lambda_{\text {th }}$ vanishes. That is, $\Phi_{\text {th }}$ contains almost surely no points since the applied thinning is so strong that all points of $\Phi$ are removed almost surely. Since this case is uninteresting, in what follows we thus consider only those functions $f$ which satisfy the integrability condition

$$
\int_{0}^{\infty} r^{d-1} f(r) \mathrm{d} r<\infty
$$

Let $f \odot f$ denote the radial self-convolution of $f$, i. e.

$$
[f \odot f](r)=\int_{\mathbb{R}^{d}} f(\|x\|) f(\|x-r \cdot v\|) \mathrm{d} x
$$

for $r \geq 0$ and $v \in \mathbb{R}^{d}$ with $\|v\|=1$, which is the $d$-dimensional convolution of $f(\|\cdot\|)$ with itself at point $r \cdot v$.

Theorem 4 (Pair correlation function). The pair correlation function $g_{\text {th }}$ of the thinned point process $\Phi_{\text {th }}$ is

$$
g_{\mathrm{th}}=(1-f)^{2} \exp (\lambda f \odot f) .
$$

The following two examples illustrate for dimension $d=2$ how certain choices of $f$ influence the second-order behavior of the resulting thinned point processes.

Example 5. Let $\lambda>0$ be arbitrary and $p_{0}=1$. Consider $\operatorname{MatI}\left[\lambda, 1, f_{a, R}\right]$ with

$$
f_{a, R}(r)= \begin{cases}1 & 0 \leq r \leq a \\ \exp \left(-\frac{r^{2}-a^{2}}{R^{2}-a^{2}}\right) & \text { otherwise }\end{cases}
$$

for $R>0$ and $a \in[0, R]$. Then it is plain to see that for any $\lambda$ and $R$ fixed the intensity of $\Phi_{\mathrm{th}}$, which is distributed according to $\operatorname{MatI}\left[\lambda, 1, f_{a, R}\right]$, is

$$
\lambda_{\mathrm{th}}=\lambda \exp \left(-\pi \lambda R^{2}\right),
$$

i.e., it does not depend on a. However, depending on the parameter a, the pair correlation function of $\Phi_{\mathrm{th}}$ shows a certain range of second-order behavior, see Figure 1 . For $a=0$ the thinning generates a pure soft-core point process, $i$. e. thinning is the stronger the closer point pairs of the initial Poisson process are but each pair distance has still non-vanishing probability. In the other direction, the Matern I hard-core model is included as the limit for $a \rightarrow R$. A mixture of both hard-and soft-core type behavior can be achieved with $a \in] 0,1[$. 

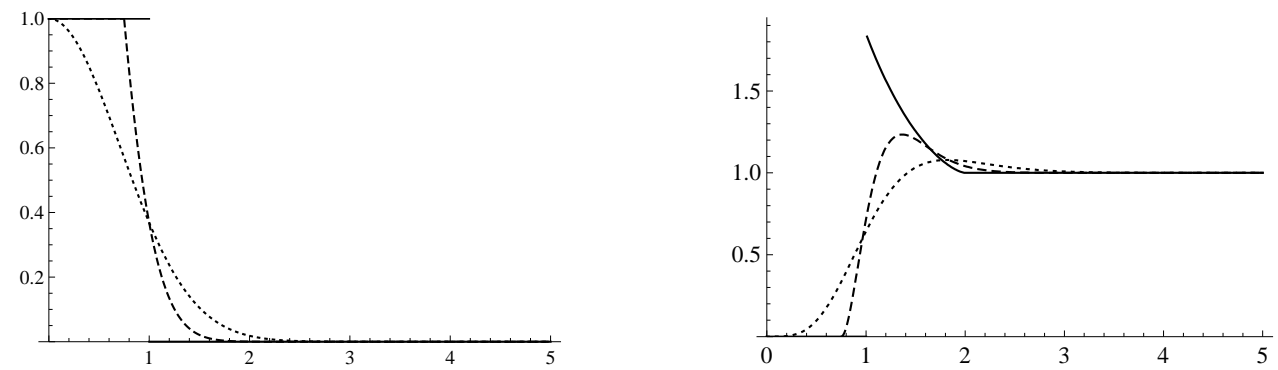

Figure 1: Left, plot of $f_{a, 1}$ for $a=0$ (dotted), $a=0.75$ (dashed) and $a=1$ (solid). Right, corresponding pair correlation functions for $\lambda=1 / 2$.
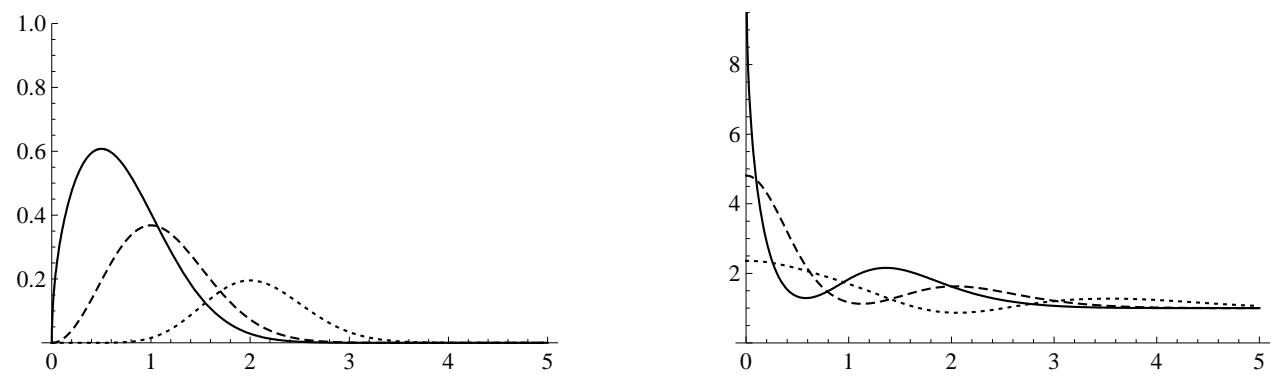

Figure 2: Left, plot of $f_{a}$ for $a=8$ (dotted), $a=2$ (dashed) and $a=0.5$ (solid). Right, corresponding pair correlation functions for $\lambda=2$.

Example 6. Another type of family $\operatorname{MatI}\left[\lambda, 1, h_{a}\right]$, where again $\lambda>0$ is arbitrary and $p_{0}=1$, is given by setting

$$
h_{a}(r)=\frac{r^{a} \exp \left(-r^{2}\right)}{\Gamma\left(1+\frac{a}{2}\right)}, \quad r \geq 0
$$

for $a \in[0, \infty[$, where $\Gamma$ denotes the $\Gamma$-function. Again, for $\lambda$ fixed, all resulting thinned point processes have the same intensity

$$
\lambda_{\mathrm{th}}=\lambda \exp (-\pi \lambda)
$$

i. e. independent from $a \geq 0$. Here, the thinning based on $h_{a}$ results in aggregation or cluster-like processes which is indicated by pair correlation functions with its maximum in the origin, see Figure 2 since points close together are deleted with relatively low probability.

Examples 5 and 6 show that the behavior of the pair correlation function of the thinned process $\Phi_{\text {th }}$ might be adjusted using appropriate functions $f$ such that it is 
possible to model soft-core, hard-core as well as aggregative point processes [cf. Illian et al. 2008. Furthermore, a 'mixture' of these types can be obtained combining the corresponding functions $f$. In summary, this reveals high flexibility of this approach with respect to second-order properties.

\section{Probabilistic thinning for marked Poisson processes}

Marked point processes as generalizations of usual point processes are highly relevant in practical applications. Many ecological and environmental systems can be described by marked point processes [see Gavrikov and Stoyan, 1995. Furthermore, there is a large literature on processes of non-overlapping grains in physics and chemistry [see Andersson et al. 2006, Månsson and Rudemo, 2002]. Here, Matérn hard-core processes are equipped with random radii as marks. While in the previous section a dependent thinning model generalizing the usual Matérn I hard-core point process was introduced, the aim of the present section is to carry over this approach to respective marked point processes. Again, intensity and pair correlation function of the corresponding unmarked point process can be given explicitly.

Let $\Psi$ be a homogeneous independently marked Poisson point process in $\mathbb{R}^{d}$ with intensity $\lambda$ and independent and identically distributed (i. i. d.) real-valued marks with $\mu$ as its mark distribution. Furthermore, let $\left.\left.p_{0} \in\right] 0,1\right]$, and $f:\left[0, \infty\left[\times \mathbb{R}^{2} \longrightarrow[0,1]\right.\right.$ be a fixed measurable function satisfying $f(\cdot, m, n)=f(\cdot, n, m)$ for all $m, n \in \mathbb{R}$.

From $\Psi$ a new model $\Psi_{\mathrm{th}}$ is derived by applying the following probabilistic dependent thinning rule to $\Psi$. The marked point $(x, m) \in \Psi$ is retained with probability

$$
p(x, m, \Psi)=p_{0} \prod_{\substack{(y, n) \in \Psi \\ y \neq x}}[1-f(\|x-y\|, m, n)]
$$

independently from deleting or retaining other marked points of $\Psi$. This means that two points of distance $r>0$ apart with marks $m$ and $n$ delete each other independently with probability $f(r, m, n)$, and, again, independently from deleting due to pairwise interaction, each surviving point is then additionally deleted with probability $1-p_{0}$.

Since the thinning rule is again independent both from location and direction, the point process $\tilde{\Psi}_{\text {th }}$ of unmarked points of $\Psi_{\text {th }}$ inherits both stationarity and isotropy from the homogeneous Poisson process of unmarked points of $\Psi$.

In the following we will sometimes write $\operatorname{MatI}\left[\lambda, \mu, p_{0}, f\right]$ for the distribution of $\Psi_{\mathrm{th}}$. Of course, if $f$ does not depend on the marks the unmarked point process $\tilde{\Psi}_{\text {th }}$ coincides with the model MatI $\left[\lambda, p_{0}, f\right]$ introduced in Section 2.1. and all formulas given there appear as particular cases of the subsequent results.

Example 7. Taking $f(r, m, n)=\mathbb{1}_{[0, m+n]}(r), r, m, n \geq 0$, and random marks uniformly from $[0,1]$, we obtain a hard-core point process with random hard-core radii, i. e., we can interpret the retained marked point $(x, m)$ as a ball of radius $m$ centered in $x$, see Figure 3. This example was studied by Månsson and Rudemo [2002] as a model for systems of varying-sized, non-overlapping spherical grains. 


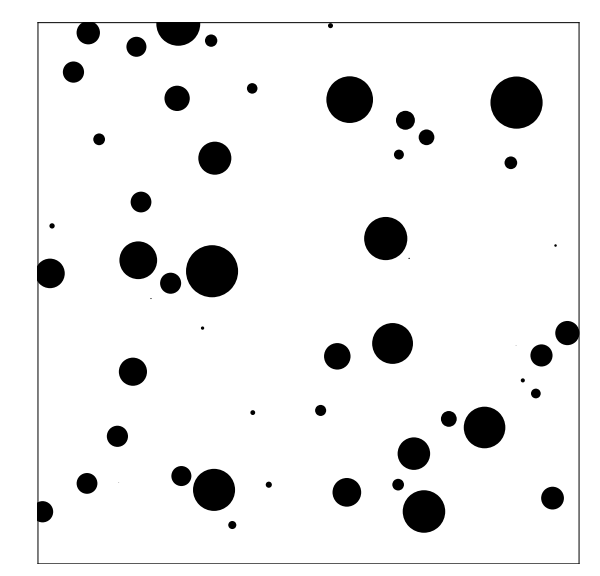

Figure 3: Sample of $\Psi_{\mathrm{th}}$ in dimension $d=2$ for $\lambda=100, p_{0}=1, f(\cdot, m, n)=\mathbb{1}_{[0, m+n]}$ and $\mu$ the uniform distribution on $[0,1]$.

Theorem 8 (Intensity). The intensity $\lambda_{\mathrm{th}}$ of the thinned point process $\tilde{\Psi}_{\mathrm{th}}$ is

$$
\lambda_{\mathrm{th}}=\lambda p_{0} \int_{\mathbb{R}} \exp \left(-\lambda d b_{d} \int_{\mathbb{R}} \int_{0}^{\infty} f(r, m, l) r^{d-1} \mathrm{~d} r \mu(\mathrm{d} l)\right) \mu(\mathrm{d} m) .
$$

As an example, consider once more the case $p_{0}=1$ and $f(\cdot, m, l)=\mathbb{1}_{[0, m+l]}$ with positive-valued mark distribution $\mu$. Then the result in Theorem 8 reads

$$
\lambda_{\text {th }}=\lambda \int_{0}^{\infty} \exp \left(-\lambda b_{d} \int_{0}^{\infty}(l+m)^{d} \mu(\mathrm{d} l)\right) \mu(\mathrm{d} m),
$$

which coincides with the formula in Månsson and Rudemo, 2002, Section 2.2, Example 2.1].

Theorem 9 (Pair correlation function). The pair correlation function of the thinned point process $\tilde{\Psi}_{\mathrm{th}}$ is

$$
g_{\mathrm{th}}(r)=\frac{\lambda^{2} p_{0}^{2}}{\lambda_{\mathrm{th}}^{2}} \int_{\mathbb{R}} \int_{\mathbb{R}}(1-f(r, m, n))^{2} q_{m} q_{n} q_{m, n}(r) \mu(\mathrm{d} m) \mu(\mathrm{d} n), \quad r \geq 0,
$$

where

$$
q_{m, n}(r)=\exp \left(\lambda \int_{\mathbb{R}} f(\cdot, m, l) \odot f(\cdot, n, l)(r) \mu(\mathrm{d} l)\right), \quad r \geq 0
$$

and

$$
q_{m}=\exp \left(-\lambda \int_{\mathbb{R}} \int_{\mathbb{R}^{d}} f(\|x\|, m, l) \mathrm{d} x \mu(\mathrm{d} l)\right) .
$$

Note that $g_{\mathrm{th}}(r)$ actually does not depend on $p_{0}$ since it appears also as a factor in $\lambda_{\text {th }}$ and thus cancels out. 


\section{Probabilistic thinning model - Matérn II case}

In the previous sections, a certain kind of dependent thinning has been introduced where two competing points of an underlying Poisson process are both removed with some probability depending on a deterministic function $f$. While this is a generalization of the classical Matérn I hard-core point process, in the present section we aim to generalize the classical Matérn II model in a similar fashion. For that purpose, weights will be assigned once to all the points and in a competition between two points only one of them, namely the point equipped with weight greater than or equal to the weight of the other point will be removed with some probability. Again, we can derive expressions for both the intensity and the pair correlation function of the resulting process of unmarked points.

Let $\Pi$ be a homogeneous independently marked Poisson point process in $\mathbb{R}^{d}$ with intensity $\lambda$ and i.i.d. $\mathbb{R}^{2}$-valued marks. The first component $m$ of any such random bivariate mark $(m, v)$ has distribution $\mu$ and the second component $v$ has distribution $\nu_{m}$ which might depend on $m$. The mark $m$ plays the same role as in Section 3 and is sometimes referred to as 'mark' whereas $v$ serves as a weight used in the thinning procedure and is thus sometimes referred to as 'weight'. Furthermore, let again $\left.\left.p_{0} \in\right] 0,1\right]$, and $f:\left[0, \infty\left[\times \mathbb{R}^{2} \longrightarrow[0,1]\right.\right.$ be a fixed measurable mapping satisfying $f(\cdot, m, n)=f(\cdot, n, m)$ for all $m, n \in \mathbb{R}$.

From $\Pi$ a new model $\Pi_{\text {th }}$ is derived by applying the following probabilistic dependent thinning rule. The point $(x, m, v) \in \Pi$ is kept as a point of $\Pi_{\mathrm{th}}$ with probability

$$
p(x, m, v, \Pi)=p_{0} \prod_{\substack{(y, n, w) \in \Pi \\ y \neq x}}[1-\mathbb{1}\{v \geq w\} f(\|x-y\|, m, n)]
$$

independently from deleting or retaining other points of $\Pi$, where $\mathbb{1}\{A\}$ is the indicator of event $A$. This means that if two points with marks $m$ and $n$ are a distance $r>0$ apart then only the point with weight greater than or equal to the weight of the other point is deleted by the other point with probability $f(r, m, n)$. Additionally, each surviving point is then again independently $p_{0}$-thinned.

In the following we will denote by $\operatorname{MatII}\left[\lambda, \mu,\left(\nu_{m}\right)_{m \in \mathbb{R}}, p_{0}, f\right]$ the distribution of $\Pi_{\mathrm{th}}$.

Note that the meaning of the weights in the thinning rule 8 is here in accordance with the meaning of the respective weights used in the definition of the original Matérn II hard-core point process in most of the literature Illian et al. , 2008, Stoyan et al. 1995. Stoyan and Stoyan, 1985. (but not [Månsson and Rudemo, 2002]), i. e., they have to be understood more (biologically) as times of appearance than importance weights, and in a competition the lower weight wins.

Remark 10. Taking $f(\cdot, m, n)=\mathbb{1}_{[0, m+n]}(\cdot), m, n \geq 0$, and marks according to a positive mark distribution $\mu$ results in a hard-core process with random hard-core radii. This case was also studied by Månsson and Rudemo [2002] as an extension of Matérn's second hard-core point process to random configurations of non-overlapping spheres. The original Matérn II point process with hard-core radius $R>0$ can be obtained using $\mu=\delta_{R}$ and $\nu_{m}$ as the uniform distribution on $[0,1]$. 
Remark 11. The particular choice $\nu_{m}=\delta_{1}$ for all marks $m$ leads back to the model $\Psi_{\text {th }}$ described in Section 3 . Here, all points would have the same weight such that two competing points delete each other independently with the same probability.

Denote by $F_{\tau}$ the cumulative distribution function of a probability measure $\tau$ on $\mathbb{R}$, i. e. $\left.\left.F_{\tau}(t)=\tau(]-\infty, t\right]\right), t \in \mathbb{R}$.

For simplicity assume in what follows that the weight distributions $\nu_{l}$ are all (absolutely) continuous (w.r.t. lebesgue measure) but may still depend on mark $l$. The main effect is that the event $\mathbb{1}\{v \geq w\}$ has probability zero and hence only one of two competing points is deleted with some probability. In particular, this excludes the Matérn-I-like processes introduced in the previous sections.

Furthermore, let us recall that the point process $\tilde{\Pi}_{\text {th }}$ of unmarked points of $\Pi_{\text {th }}$ inherits both stationarity and isotropy from the homogeneous Poisson process of unmarked points of $\Pi$.

Theorem 12 (Intensity). The intensity $\lambda_{\mathrm{th}}$ of the thinned point process $\tilde{\Pi}_{\mathrm{th}}$ is

$$
\lambda_{\mathrm{th}}=\lambda p_{0} \int_{\mathbb{R}} \int_{\mathbb{R}} q_{m}(w) \nu_{m}(\mathrm{~d} w) \mu(\mathrm{d} m),
$$

where

$$
q_{m}(w)=\exp \left(-\lambda \int_{\mathbb{R}} F_{\nu_{l}}(w) \int_{\mathbb{R}^{d}} f(\|x\|, m, l) \mathrm{d} x \mu(\mathrm{d} l)\right) .
$$

In the special case that the weight distribution $\nu_{l}$ does not depend on the mark $l$, i. e., $\nu_{l}=\nu$ for some continuous distribution $\nu$, Theorem 12 simplifies to

$$
\begin{aligned}
\lambda_{\mathrm{th}} & =\lambda p_{0} \int_{\mathbb{R}} \int_{\mathbb{R}} \exp \left(-\lambda F_{\nu}(w) \int_{\mathbb{R}} \int_{\mathbb{R}^{d}} f(\|x\|, m, l) \mathrm{d} x \mu(\mathrm{d} l)\right) \nu(\mathrm{d} w) \mu(\mathrm{d} m) \\
& =\lambda p_{0} \int_{\mathbb{R}} \int_{0}^{1} \exp \left(-\lambda u \int_{\mathbb{R}} \int_{\mathbb{R}^{d}} f(\|x\|, m, l) \mathrm{d} x \mu(\mathrm{d} l)\right) \mathrm{d} u \mu(\mathrm{d} m) \\
& =p_{0} \int_{\mathbb{R}} \frac{1-\exp \left(-\lambda \int_{\mathbb{R}} \int_{\mathbb{R}^{d}} f(\|x\|, m, l) \mathrm{d} x \mu(\mathrm{d} l)\right)}{\int_{\mathbb{R}} \int_{\mathbb{R}^{d}} f(\|x\|, m, l) \mathrm{d} x \mu(\mathrm{d} l)} \mu(\mathrm{d} m),
\end{aligned}
$$

by change of variables. In the case $p_{0}=1$ and $f(\cdot, m, n)=\mathbb{1}_{[0, m+n]}$ formula $10 p$ coincides with the result stated in Månsson and Rudemo, 2002, Theorem 3.1].

Remark 13. ¿From Equation (9) it is easy to see that, due to $F_{\nu_{l}}(w) \leq 1$ for all $w$, the intensity of the thinned point process $\Pi_{\text {th }}$ is always greater than the intensity of a thinned point process according to $\operatorname{MatI}\left[\lambda, \mu, p_{0}, f\right]$ from Section 3 with the same parameters.

Theorem 14 (Pair correlation function). The pair correlation function $g_{\mathrm{th}}$ of the thinned point process $\tilde{\Pi}_{\mathrm{th}}$ is

$$
g_{\mathrm{th}}(r)=\frac{\lambda^{2} p_{0}^{2}}{\lambda_{\mathrm{th}}^{2}} \int_{\mathbb{R}} \int_{\mathbb{R}}(1-f(r, m, n)) I_{r}(m, n) \mu(\mathrm{d} m) \mu(\mathrm{d} n), \quad r>0,
$$


where

$$
I_{r}(m, n)=\int_{\mathbb{R}} \int_{\mathbb{R}} q_{m}(w) q_{n}(t) q_{m, n}(w, t, r) \nu_{m}(\mathrm{~d} w) \nu_{n}(\mathrm{~d} t)
$$

with $q_{m}(w)$ from Equation 9 and

$$
q_{m, n}(w, t, r)=\exp \left(\lambda \int_{\mathbb{R}} F_{\nu_{l}}(\min \{w, t\})[f(\cdot, m, l) \odot f(\cdot, n, l)](r) \mu(\mathrm{d} l)\right) .
$$

Again $g_{\mathrm{th}}(r)$ does not depend on $p_{0}$. In the special case where $\nu_{l}=\nu$ does not depend on $l, I_{r}(m, n)$ can be written as

$$
I_{r}(m, n)=\int_{0}^{1} \int_{0}^{1} q_{m}^{s} q_{n}^{t} q_{m, n}(r)^{\min \{s, t\}} \mathrm{d} s \mathrm{~d} t=J(a, b, c)+J(b, a, c)
$$

where

$$
J(a, b, c)=\frac{1}{b(a+b+c)}+\frac{1}{a+c}\left(\frac{\exp (a+b+c)}{a+b+c}-\frac{\exp (b)}{b}\right)
$$

with $q_{m}$ and $q_{m, n}(r)$ according to Equation (7) and Equation (6), respectively, and $a=\log q_{m}, b=\log q_{n}$ and $c=\log q_{m, n}(r)$.

\section{Applications}

\subsection{Fontainebleau sandstone}

The first data set is a point pattern describing the pore network of a sample of Fontainebleau sandstone. A visualization is given in Figure 4 A detailed description how this point pattern was obtained can be found in [Sok et al. 2002. It has been further analyzed in the literature, for instance, Tscheschel and Stoyan [2003] discuss second-order characteristics and a certain Euler-Poincaré characteristic connected with the data. A standard test of the hypothesis that the pattern is of CSR type (complete spatial randomness) Illian et al. 2008 results in rejection with a $p$-value of 0.0002 .

The minimum interpoint distance in the pattern is $60.7 \mu \mathrm{m}$, and it is just this hardcore distance which leads to a rejection of the CSR hypothesis. Consequently, a hardcore point process model seems to be more appropriate for this data. Because of the low point density Matérn-like point processes are promising.

The plot in Figure 4 shows the estimated pair correlation function $\hat{g}$ [see Illian et al. 2008. Section 4.3.3] of the data and the pair correlation function $g$ both of a fitted Matérn I and a fitted Matérn II hard-core point process.

Taking the minimum interpoint distance of $60.7 \mu \mathrm{m}$ as an estimate for the hard-core distance $R$ (which is even a maximum likelihood estimate), fitting is here easily done by estimating the intensity $\lambda$ of the underlying Poisson process as the only remaining unknown parameter by the method of moments. That is, due to

$$
\lambda_{I}=\lambda \exp \left(-\lambda b_{3} R^{3}\right), \quad \lambda_{I I}=\frac{1-\exp \left(-\lambda b_{3} R^{3}\right)}{b_{3} R^{3}},
$$



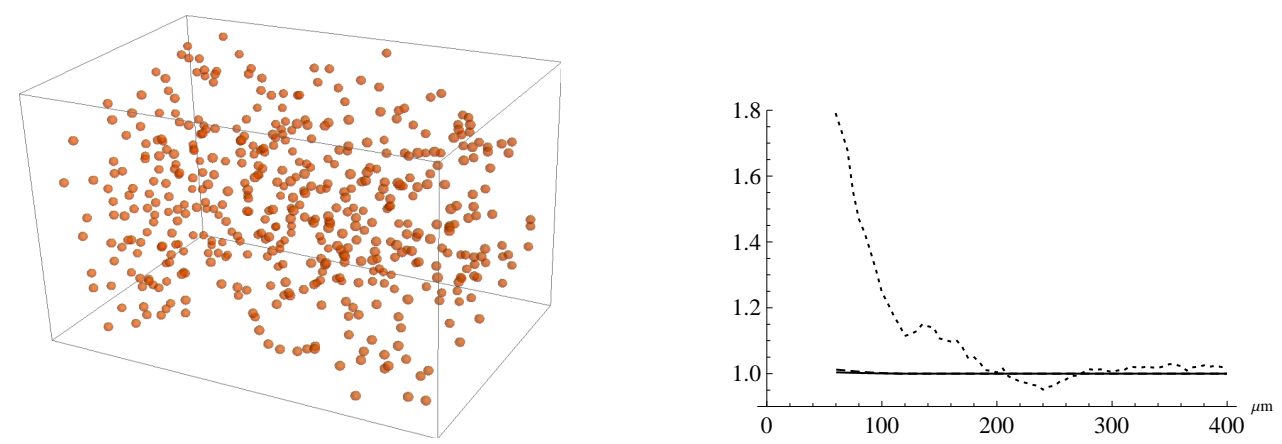

Figure 4: Left, 470 nodes of a network adapted to the pores of a $2 \times 2 \times 3 \mathrm{~mm}$ sample of Fontainebleau sandstone. Right, the empirical pcf $\hat{g}$ (dotted), $g$ for Matérn I (dashed) and for Matérn II (solid line).

for the intensities of the, respectively, Matérn I and Matérn II hard-core point process, an estimate of $\lambda$ can be obtained by solving for $\lambda$ in the equations $\hat{\lambda}=\lambda_{I}$ and $\hat{\lambda}=\lambda_{I I}$, respectively, where $\hat{\lambda}=39.17 \mathrm{~mm}^{-3}$ is the empirical intensity of the data. However, Figure 4 shows clear differences between the respective pair correlation functions indicating that none of the both Matérn hard-core point processes is a good model.

Since pure hard-core point process models of Matérn type seemed to be not appropriate we have then fitted a model MatI $\left[\lambda, p_{0}, f_{R, a, b}\right]$ from Section 2.1 with

$$
f_{R, a, b}(r)= \begin{cases}1 & 0 \leq r \leq R \\ \frac{1}{a} \exp \left(-\frac{(r-R)^{2}}{b}\right) & \text { otherwise }\end{cases}
$$

with $R=60.7 \mu \mathrm{m}$. Here, as parameter estimate the best possible choice of $\left(\lambda, p_{0}, a, b\right)$ was taken, meant in the sense that under the condition $\lambda_{\text {th }}=\hat{\lambda}$ the contrast

$$
\Delta\left(\lambda, p_{0}, a, b\right)=\int_{r_{\min }}^{r_{\max }}\left[\hat{g}(r)-g_{\mathrm{th}}(r)\right]^{2} \mathrm{~d} r
$$

is minimized with respect to $\left(\lambda, p_{0}, a, b\right)$, where $\left[r_{\min }, r_{\max }\right]$ is a suitable domain. This is a variant of the well-known minimum contrast method for parameter estimation Diggle, 2003, Heinrich, 1992 where here the difference to be minimized depends on the pair correlation function as that summary statistics which is available at least via numerical integration for the models under consideration. The minimum contrast method using the pair correlation function has been also successfully applied by Stoyan and Stoyan [1996 and Møller and Waagepetersen [2003, p. 183]. This resulted in estimates $\lambda=1919 \mathrm{~mm}^{-3}, p_{0}=0.92, a=6.3$ and $b=3917 \mu \mathrm{m}^{2}$. Figure 5 shows the estimated function $f_{R, a, b}$ as well as both the empirical pair correlation function $\hat{g}$ of the network data and the pair correlation function $g_{\text {th }}$ of the estimated model. The visual 

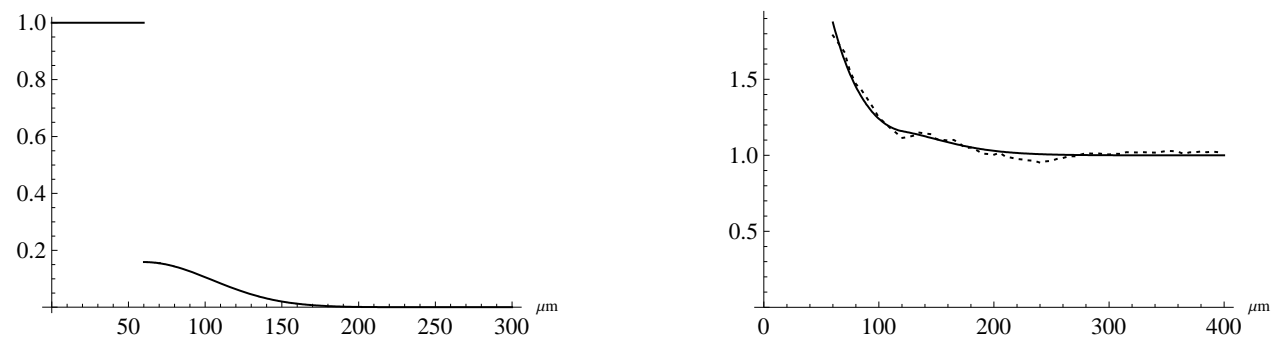

Figure 5: Left, plot of $f_{R, a, b}, R=60.7 \mu \mathrm{m}, a=6.3$ and $b=3917 \mu \mathrm{m}^{2}$. Right, pair correlation functions $\hat{g}$ (dotted) of the network data and $g_{\text {th }}$ (solid line) of the fitted model.
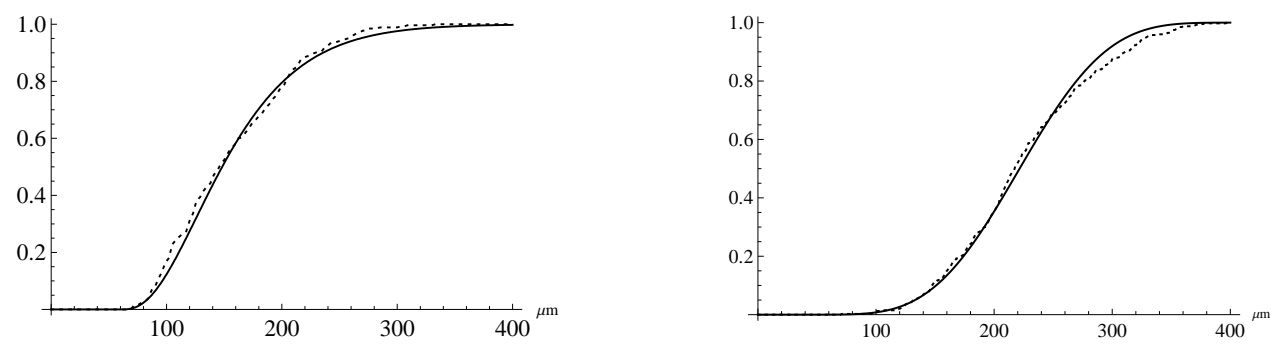

Figure 6: Left, empirical nearest-neighbor distribution function of the network data (dotted) and nearest-neighbor distribution function of the fitted model (solid). Right, same for empirical empty space function.

finding from Figure 5 (right) that the fit is good can be suggested by formal tests. For instance, a deviation test [see Illian et al., 2008, Section 7.4] for the corresponding $L$-functions Illian et al. 2008, Section 4.3] with global deviation measure

$$
\Delta=\int_{0}^{\max }\left|\hat{L}(r)-L_{\mathrm{th}}(r)\right|^{2} \mathrm{~d} r
$$

( $p$-value 0.18 ) with $k=99$ simulations indicates that now the model was chosen flexible enough to give a fit which mimics the second-order behavior of the data sufficiently well. Two other deviation tests with $k=99$ simulations using the nearest-neighbor distance distribution function as well as the empty space function (or 'spherical contact distribution function') Illian et al. 2008, Section 4.2] (see Figure 6 for plots) instead of the $L$-function then show that the fit is good also in other respects ( $p$-values 0.14 and 0.12 , respectively). 


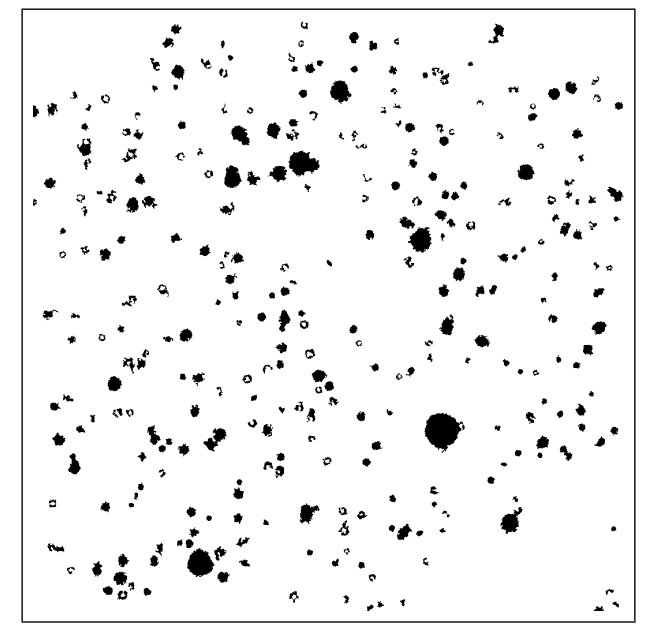

Figure 7: One of the used QICPIC images, size $1 \mathrm{~m} \times 1 \mathrm{~m}$.

\subsection{Patterns of deagglomerated alumina particles}

The second data set are three samples of a mono-layer of deagglomerated alumina particles within water which serve as a starting point for the investigation of certain agglomeration processes not discussed here. The patterns, one shown in Figure 7. were obtained with a QICPIC sensor (Sympatec/Germany), which is a measurement device for dynamic picture analysis. For the test setup a liquid dispersing unit was used to get such a mono-layer flow of deagglomerated alumina particles through a flat cuvette where then the images were recorded. The median of the alumina particles is approximately $10 \mu \mathrm{m}$. Due to the recording process, some of the particles look like open circles. Although they are all non-overlapping in space, some particles close together appear to be connected due to the projective nature of the recording.

The planar pattern of particles is quite sparse for which reason it might be modeled by a thinned marked Poisson process as in Sections 3 and 4 However, a Matérn-IItype model from Section 4 might be comparatively more promising due to the higher attainable intensities, see Remark 13 .

Our first attempt was to fit a Matérn II process for hard spheres as in Remark 10 with gamma-distributed radius marks, where for practical reasons the distribution was truncated at some high value. The distribution of the weight marks was chosen to be the uniform distribution on $[0,1]$. Here, three parameters, $\left(\lambda=315 \mathrm{~mm}^{-2}\right.$, shape $=6.5$, rate $=0.00128 \mathrm{~mm})$, were estimated again by the minimum contrast method using the pair correlation function. A comparison of the resulting model pair correlation function $g_{M}$ and the empirical pair correlation function $\hat{g}$ of the data shown in Figure 8 indicates that this kind of model is not flexible enough already for the second-order behavior of the data. This is supported also by the visual inspection of the corresponding ('Matérn') nearest-neighbor distance distribution function and empty space function, 

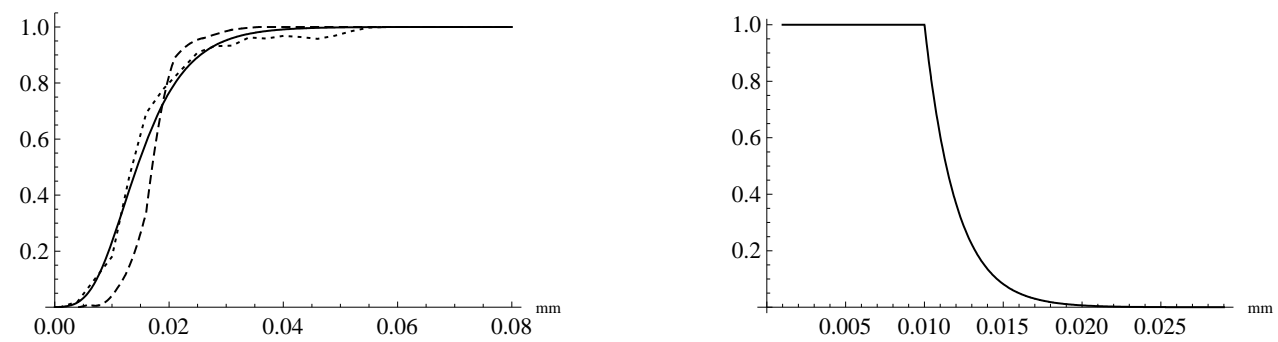

Figure 8: Plot of $\hat{g}$ (dotted), $g_{M}$ (dashed) $g_{\text {th }}$ (solid), left. Function $f_{c}$ for fixed $c$ and $m, n$.

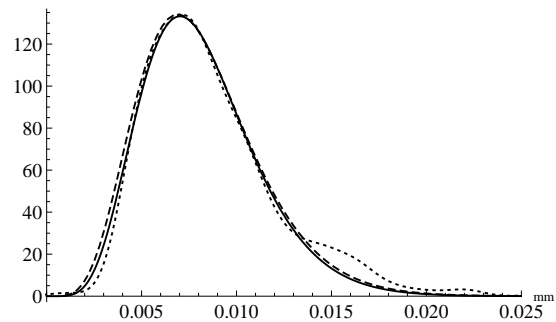

Figure 9: Empirical p.d.f. of radii of the data (dotted), Matérn (dashed) and $\Pi_{\text {th }}$ (solid).

respectively, in Figure 10, see also Table 1 for several related deviation tests.

This motivated us to try modeling with the generalization introduced in Section 4 Here, the ansatz is $\Pi_{\mathrm{th}} \sim \operatorname{MatII}\left[\lambda, \Gamma(\alpha, \beta)\right.$, Uniform $\left.[0,1], p_{0}, f_{c}\right]$ with

$$
f_{c}(r, m, n)= \begin{cases}1 & 0 \leq r \leq m+n \\ \exp [-c(r-m-n)] & \text { otherwise }\end{cases}
$$

(shown in Figure 8 and $\Gamma(\cdot, \cdot)$ as gamma distribution.

Parameter estimation by the minimum contrast method (11) with the pair correlation function yielded estimates $\lambda=335 \mathrm{~mm}^{-2}, \alpha=6.3, \beta=0.00127 \mathrm{~mm}, p_{0}=0.96$ and $c=119 \mathrm{~mm}^{-1}$. The resulting characteristics like the pair correlation function $g_{\mathrm{th}}$ shown in Figures 8 to 10 indicate a much better fit than the model of the first attempt. This is also supported by the corresponding deviation tests, see Table 1, each based on $k=99$ simulations of the fitted model.

\section{Conclusions and outlook}

In this paper, we have examined a new class of point processes generalizing the Matérn I and II hard-core point processes as well as the independent thinning approach. Clearly, 

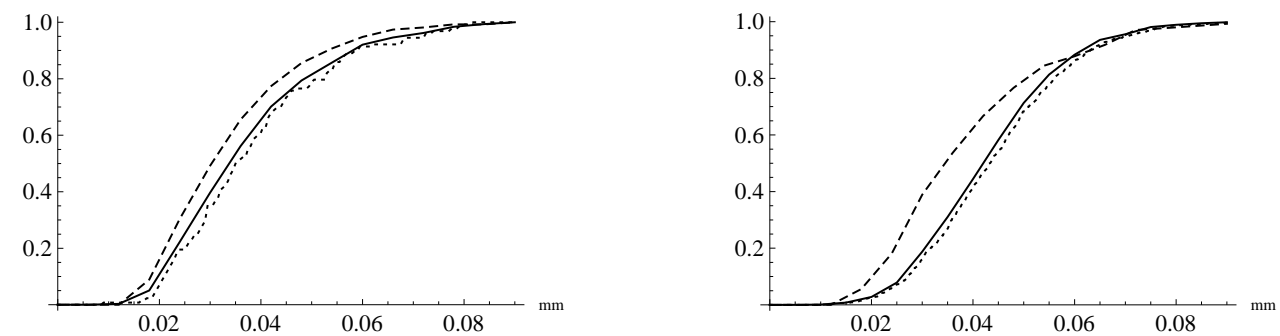

Figure 10: Left, empirical c.d.f. of the nearest-neighbor distance of the data (dotted), Matérn (dashed) and $\Pi_{\text {th }}$ (solid). Right, same for empirical empty space function.

\begin{tabular}{l|c|c|c|c} 
& pcf & p.d.f radii & nearest-n. & empty sp. \\
\hline Matérn & 0.00 & 0.13 & 0.01 & 0.00 \\
$\Pi_{\text {th }}$ & 0.16 & 0.17 & 0.07 & 0.06 \\
\hline
\end{tabular}

Table 1: $p$-values for the deviation tests of the models 'Matérn' and $\Pi_{\text {th }}$ using the pair correlation function (pcf), the probability density function of the radius marks after thinning (p.d.f radii), the nearest neighbor distance distribution function (nearest-n.) and the empty space function (empty sp.), each based on 99 simulations. 
the proposed new model is not suited for very dense and structured packings of particles, since, like for the Matérn processes, there is a relatively small upper bound for the intensity for any given $f$ not vanishing almost everywhere. However, it provides a flexible and simple to fit model in the class of dependent point process models. Unlike for the also very flexible Gibbs point processes the simple mathematical structure allows for a simple and straight forward simulation and a direct computation of structure functions of the resulting point process, also simplifying the application of standard fitting procedures. The approach allows for a interpretable descriptions of interactions, like interaction of shaped objects or non-deterministic death from competition. In our further research we also found that this class corresponds to distributions derived for snapshot point patterns in moving particle systems models we developed for the alumina particles. However, this relation has to be discussed in a separate article introducing these moving particle systems modeling.

Thus we think that this new class is worth considering for modeling various real world point patterns and systems of particle centers.

\section{Proofs}

\subsection{Proof of Theorem 8}

The intensity measure Daley and Vere-Jones, 1988, Illian et al., 2008, Møller and Waagepetersen, 2003, Stoyan et al. $1995, \alpha_{\text {th }}$ of $\Psi_{\text {th }}$ satisfies

$$
\alpha_{\mathrm{th}}(\mathrm{d}(x, m))=p_{0} q_{m} \alpha_{\Psi}(\mathrm{d}(x, m))
$$

where $\alpha_{\Psi}(\mathrm{d}(x, m))=\lambda \mathrm{d} x \mu(\mathrm{d} m)$ is the intensity measure of $\Psi$ and $q_{m}$ is the probability that a primary point with mark $m$ in $\Psi$ is retained as secondary event in $\Psi_{\text {th }}$ when thinning would be restricted to pairwise interaction, i. e., (1) would be applied with $p_{0}=1$.

Using Palm theory Daley and Vere-Jones, 1988, Illian et al., 2008, Stoyan et al. 1995 and stationarity of $\Psi, q_{m}$ is the probability that under the reduced Palm distribution $P_{(o, m)}^{!}$of $\Psi$, where $o$ denotes the origin, the point $(o, m)$ is not deleted by any other point. Since $\Psi$ is a marked Poisson point process this is, due to the SlivnyakMecke theorem [Illian et al., 2008, Møller and Waagepetersen, 2003, equivalent to the probability that the point $(o, m)$ is not deleted by any point from $\Psi$ when the same thinning rule is applied.

Let $Q_{m}$ be the marked point process which consists of all points from $\Psi$ causing a deletion of $(o, m)$. Then $q_{m}$ is simply the probability that $Q_{m}$ has no points. Obviously, $Q_{m}$ is obtained by independent thinning of $\Psi$, i. e.

$$
Q_{m}=\sum_{(x, l) \in \Psi} \beta(x, m, l) \delta_{(x, l)}
$$

where $\beta(x, m, l)$ is Bernoulli-distributed with parameter $f(\|x\|, m, l)$ and $\delta_{(x, l)}$ denotes the Dirac measure centered on $(x, l)$. Hence, $Q_{m}$ is an inhomogeneous marked Poisson 
process [Illian et al. 2008, Section 6.1] with intensity measure

$$
\alpha_{Q_{m}}(\mathrm{~d}(x, l))=f(\|x\|, m, l) \alpha_{\Psi}(\mathrm{d}(x, l)=f(\|x\|, m, l) \lambda \mathrm{d} x \mu(\mathrm{d} m) .
$$

Since $q_{m}$ is the void probability of the Poisson process $Q_{m}$ this implies

$$
\begin{aligned}
q_{m} & =\mathbb{P}\left(Q_{m}\left(\mathbb{R}^{d} \times \mathbb{R}\right)=0\right)=\exp \left(-\alpha_{Q_{m}}\left(\mathbb{R}^{d} \times \mathbb{R}\right)\right) \\
& =\exp \left(-\lambda \int_{\mathbb{R}} \int_{\mathbb{R}^{d}} f(\|x\|, m, l) \mathrm{d} x \mu(\mathrm{d} l)\right) \\
& =\exp \left(-\lambda d b_{d} \int_{\mathbb{R}} \int_{0}^{\infty} f(r, m, l) r^{d-1} \mathrm{~d} r \mu(\mathrm{d} l)\right)
\end{aligned}
$$

using polar coordinates in the last step. Hence, due to Equation (12), the point process of unmarked points of $\Psi_{\text {th }}$ has intensity

$$
\begin{aligned}
\lambda_{\mathrm{th}} & =\lambda p_{0} \int_{\mathbb{R}} q_{m} \mu(\mathrm{d} m) \\
& =\lambda p_{0} \int_{\mathbb{R}} \exp \left(-\lambda d b_{d} \int_{\mathbb{R}} \int_{0}^{\infty} f(r, m, l) r^{d-1} \mathrm{~d} r \mu(\mathrm{d} l)\right) \mu(\mathrm{d} m) .
\end{aligned}
$$

\subsection{Proof of Theorem 9}

Let $\kappa_{m, n}(r)$ be the probability that two points in $\Psi$ with mark $m$ and $n$ a distance $r$ apart are both retained in $\Psi_{\text {th }}$ when (4) is applied with $p_{0}=1$. Then the second-order factorial moment measure Daley and Vere-Jones, 1988, Illian et al., 2008, Møller and Waagepetersen, 2003. Stoyan et al. 1995 $\alpha_{\mathrm{th}}^{(2)}$ of $\Psi_{\mathrm{th}}$ satisfies

$$
\alpha_{\text {th }}^{(2)}(\mathrm{d}(x, m, y, n))=p_{0}{ }^{2} \kappa_{m, n}(\|x-y\|) \alpha_{\Psi}^{(2)}(\mathrm{d}(x, m, y, n)),
$$

where the second-order factorial moment measure $\alpha_{\Psi}^{(2)}$ of $\Psi$ factorizes to

$$
\alpha_{\Psi}^{(2)}(\mathrm{d}(x, m, y, n))=\alpha_{\Psi}(\mathrm{d}(x, m)) \alpha_{\Psi}(\mathrm{d}(y, n))=\lambda^{2} \mathrm{~d} x \mu(\mathrm{d} m) \mathrm{d} y \mu(\mathrm{d} n)
$$

since $\Psi$ is a Poisson process [see Daley and Vere-Jones, 1988]. Using again Palm theory and the Slivnyak-Mecke theorem, $\kappa_{m, n}(r)$ equals the probability that the two points $(o, m)$ and $(z, n),\|z\|=r$, do not delete each other and are non of them is deleted by any point from $\Psi$ according to the thinning rule (4) with $p_{0}=1$. Since both events are independent and the probability of the first event is $(1-f(r, m, n))^{2}$, it follows

$$
\kappa_{m, n}(r)=(1-f(r, m, n))^{2} \mathbb{P}\left(W_{z, m, n}\left(\mathbb{R}^{d} \times \mathbb{R}\right)=0\right),
$$

where $W_{z, m, n}$ is the marked point process which consists of all points from $\Psi$ causing a deletion of $(o, m)$ or $(z, n)$. Due to independent thinning, i. e.,

$$
W_{z, m, n}=\sum_{(x, l) \in \Psi} \max \left\{\gamma_{0}(x, m, l), \gamma_{r}(x, n, l)\right\} \delta_{(x, l)},
$$


where $\gamma_{r}(x, a, b)$ is Bernoulli-distributed with parameter $f(\|x-r \cdot v\|, a, b),\|v\|=1$, $W_{z, m, n}$ is an inhomogeneous marked Poisson process with intensity measure

$$
\begin{aligned}
\alpha_{W_{z, m, n}}(\mathrm{~d}(x, l))= & {[f(\|x\|, m, l)+f(\|x-r \cdot v\|, n, l)-f(\|x\|, m, l) f(\|x-r \cdot v\|, n, l)] } \\
& \times \lambda \mathrm{d} x \mu(\mathrm{d} l) .
\end{aligned}
$$

According to Equation (16), this yields

$$
\begin{aligned}
\kappa_{m, n}(r)=(1 & f(r, m, n))^{2} \exp \left(-\alpha_{W_{z, m, n}}\left(\mathbb{R}^{d} \times \mathbb{R}\right)\right) \\
=(1 & -f(r, m, n))^{2} \exp \left(-\lambda \int_{\mathbb{R}} \int_{\mathbb{R}^{d}} f(\|x\|, m, l) \mathrm{d} x \mu(\mathrm{d} l)\right) \\
& \times \exp \left(-\lambda \int_{\mathbb{R}} \int_{\mathbb{R}^{d}} f(\|x\|, n, l) \mathrm{d} x \mu(\mathrm{d} l)\right) \\
& \quad \times \exp \left(\lambda \int_{\mathbb{R}} \int_{\mathbb{R}^{d}} f(\|x\|, m, l) f(\|x-r \cdot v\|, n, l) \mathrm{d} x \mu(\mathrm{d} l)\right) \\
= & (1-f(r, m, n))^{2} q_{m} q_{n} \exp \left(\lambda \int_{\mathbb{R}} f(\cdot, m, l) \odot f(\cdot, n, l)(r) \mu(\mathrm{d} l)\right),
\end{aligned}
$$

using Equation (13) and the radial convolution Equation (3) in the last step. Abbreviating the last factor of the product in Equation (17) by $q_{m, n}(r)$ and combining equations Equation (14) and Equation (15), the second-order product density of the point process $\tilde{\Psi}_{\text {th }}$ of unmarked points of $\Psi_{\text {th }}$ is

$$
\varrho_{\mathrm{th}}(r)=\lambda^{2} p_{0}^{2} \int_{\mathbb{R}} \int_{\mathbb{R}}(1-f(r, m, n))^{2} q_{m} q_{n} q_{m, n}(r) \mu(\mathrm{d} m) \mu(\mathrm{d} n) .
$$

Due to $g_{\text {th }}(r)=\varrho_{\text {th }}(r) / \lambda_{\text {th }}^{2}$ for $r \geq 0$ [llian et al. 2008 and Theorem 8 this yields the asserted form of the pair correlation function $g_{\mathrm{th}}$, i. e., in particular, $p_{0}^{2}$ cancels out.

\subsection{Proof of Theorem 12}

Basically, the idea of the proof is the same as in Section 7.1 Here, let $q_{m}(w)$ be the probability that the point $(o, m, w)$ is not deleted by any point from $\Pi$ when the thinning rule (8) with $p_{0}=1$ is applied. Then $p_{0} q_{m}(w)$ is the density of the intensity measure of $\Pi_{\mathrm{th}}$ with respect to the intensity measure $\alpha_{\Pi}$ of $\Pi, \alpha_{\Pi}(\mathrm{d}(x, m, w))=$ $\lambda \mathrm{d} x \nu_{m}(\mathrm{~d} w) \mu(\mathrm{d} m)$, and $q_{m}(w)$ equals the probability that the marked point process $Q_{m, w}$ consisting of all points from $\Pi$ causing a deletion of $(o, m, w)$ is empty. Since $Q_{m, w}$ is a Poisson process with intensity measure $\mathbb{1}\{v \leq w\} f(\|x\|, m, l) \alpha_{\Pi}(\mathrm{d}(x, l, v))$ this yields

$$
\begin{aligned}
q_{m}(w) & =\mathbb{P}\left(Q_{m, w}\left(\mathbb{R}^{d} \times \mathbb{R} \times \mathbb{R}\right)=0\right) \\
& =\exp \left(-\lambda \int_{\mathbb{R}} \int_{\mathbb{R}} \int_{\mathbb{R}^{d}} \mathbb{1}\{v \leq w\} f(\|x\|, m, l) \mathrm{d} x \nu_{l}(\mathrm{~d} v) \mu(\mathrm{d} l)\right) \\
& =\exp \left(-\lambda d b_{d} \int_{\mathbb{R}} \int_{0}^{\infty} F_{\nu_{l}}(w) f(r, m, l) r^{d-1} \mathrm{~d} r \mu(\mathrm{d} l)\right)
\end{aligned}
$$


and, finally,

$$
\lambda_{\mathrm{th}}=\lambda p_{0} \int_{\mathbb{R}} \int_{\mathbb{R}} q_{m}(w) \nu_{m}(\mathrm{~d} w) \mu(\mathrm{d} m) .
$$

\subsection{Proof of Theorem 14}

The main arguments of the proof of Theorem 9 in Section 7.2 can be carried over. Let $p_{0}^{2} \kappa_{m, n}(w, t, r)$ be the probability that two points in $\Pi$ with marks $m$ and $n$ and weights $w$ and $t$ a distance $r$ apart are both retained in $\Pi_{\mathrm{th}}$. Then $\kappa_{m, n}(w, t, r)$ equals the probability that (a) the two points $(o, m, w)$ and $(z, n, t),\|z\|=r$, do not delete each other and (b) non of them is deleted by any point from $\Pi$ according to the thinning rule (8) with $p_{0}=1$. Again, both events are independent, and the probability of event (a) is

$$
1-[\mathbb{P}(A)+\mathbb{P}(B)-\mathbb{P}(A \cap B)]=1-f(r, m, n)+\mathbb{1}\{t=w\}\left[f(r, m, n)^{2}-f(r, m, n)\right]
$$

since the probabilities that (A) $(z, n, t)$ deletes $(o, m, w)$, that (B) $(o, m, w)$ deletes $(z, n, t)$, and that both delete each other are $\mathbb{1}\{t \geq w\} f(r, m, n), \mathbb{1}\{t \leq w\} f(r, m, n)$, and $\mathbb{1}\{t=w\} f(r, m, n)^{2}$, respectively. The probability of event (b) is the probability that the Poisson process $W_{z, m, n, w, t}$ consisting of all points of $\Pi$ causing a deletion of $(o, m, w)$ or $(z, n, t)$ is empty. Hence, using $q_{m}(w)$ from 18$)$ as shorthand, it equals

$$
\mathbb{P}\left(W_{z, m, n, w, t}\left(\mathbb{R}^{d} \times \mathbb{R} \times \mathbb{R}\right)=0\right)=q_{m}(w) q_{n}(t) q_{m, n}(w, t, r),
$$

where

$$
q_{m, n}(w, t, r)=\exp \left(\lambda \int_{\mathbb{R}} \int_{\mathbb{R}^{d}} F_{\nu_{l}}(\min \{w, t\}) f(\|x\|, m, l) f(\|x-r \cdot v\|, n, l) \mathrm{d} x \mu(\mathrm{d} l)\right),
$$

since $W_{z, m, n, w, t}$ has intensity measure

$$
\begin{aligned}
& {[\mathbb{1}\{u \leq w\} f(\|x\|, m, l)+\mathbb{1}\{u \leq t\} f(\|x\|, n, l)} \\
& \quad-\mathbb{1}\{u \leq w\} f(\|x\|, m, l) \mathbb{1}\{u \leq t\} f(\|x\|, n, l)] \alpha_{\Pi}(\mathrm{d}(x, l, u))
\end{aligned}
$$

and

$$
\int_{\mathbb{R}} \mathbb{1}\{u \leq w\} \mathbb{1}\{u \leq t\} \nu_{l}(\mathrm{~d} u)=F_{\nu_{l}}(\min \{w, t\}) .
$$

Therefore, the second-order product density of $\tilde{\Pi}_{t h}$ is

$$
\begin{aligned}
\varrho_{\mathrm{th}}(r) & =\lambda^{2} p_{0}^{2} \int_{\mathbb{R}} \int_{\mathbb{R}} \int_{\mathbb{R}} \int_{\mathbb{R}} \kappa_{m, n}(w, t, r) \nu_{m}(\mathrm{~d} w) \nu_{n}(\mathrm{~d} t) \mu(\mathrm{d} m) \mu(\mathrm{d} n) \\
& =\lambda^{2} p_{0}^{2} \int_{\mathbb{R}} \int_{\mathbb{R}}(1-f(r, m, n)) I_{r}(m, n) \mu(\mathrm{d} m) \mu(\mathrm{d} n)
\end{aligned}
$$

where

$$
I_{r}(m, n)=\int_{\mathbb{R}} \int_{\mathbb{R}} q_{m}(w) q_{n}(t) q_{m, n}(w, t, r) \nu_{m}(\mathrm{~d} w) \nu_{n}(\mathrm{~d} t) .
$$

Note that the summand $\mathbb{1}\{t=w\}\left(f(r, m, n)^{2}-f(r, m, n)\right)$ in the first factor of the integrand has been left out since its integral vanishes due to the assumed continuity of the distributions $\nu_{m}$ and $\nu_{n}$. 


\section{Acknowledgments}

The authors would like to thank the German Science Foundation (DFG) for supporting the scientific work within the framework of the Collaborative Research Centre "Multi-Functional Filters for Metal Melt Filtration - A Contribute towards Zero Defect Materials" (SFB 920). They are very grateful to J. Fritzsche and F. Heuzeroth for providing their particle data sets and to D. Stoyan for inspiring discussions on the topic.

\section{References}

D. Stoyan, W. S. Kendall, and J. Mecke. Stochastic Geometry and its Applications. Wiley, Chichester, 2nd edition, 1995.

B. Matérn. Spatial variation. stochastic models and their application to some problems in forest surveys and other sampling investigations. Medd. Statens Skogsforskningsinst., 49(5):1-144, 1960.

B. Matérn. Spatial Variation. Lecture Notes in Statistics 36, Springer, New York, 1986.

N. Picard, M. Kouyate, and H. Dessard. Tree density estimations using a distance method in Mali savanna. Forest Science, 51(1):7-18, 2005.

D. Stoyan. Statistical analysis of spatial point processes: a soft-core model and cross correlations of marks. Biometrical J., 29:971-980, 1987.

W. G. Warren. Point processes in forestry. In P. S. W. Lewis, editor, Stochastic Point Processes, pages 801-816. Wiley, New York, 1972.

F. Baccelli and B. Błaszczyszyn. Stochastic Geometry and Wireless Networks, Volume II - Applications, volume 4, No 1-2 of Foundations and Trends in Networking. NoW Publishers, 2009.

A. Busson and G. Chelius. Point processes for interference modeling in CSMA/CA ad hoc networks. In Sixth ACM International Symposium on Performance Evaluation of Wireless Ad Hoc, Sensor, and Ubiquitous Networks (PE-WASUN 09), 2009.

M. Haenggi. Mean interference in hard-core wireless networks. IEEE Communications Letters, 15(8):792-794, 2011.

D. Stoyan. Thinnings of point processes and their use in the statistical analysis of a settlement pattern with deserted tillages. Statistics, 19:45-56, 1988.

J. Illian, A. Penttinen, H. Stoyan, and D. Stoyan. Statistical Analysis and Modelling of Spatial Point Patterns. Wiley, Chichester, 2008.

J. L. Batista and A. D. Maguire. Modeling the spatial structure of tropical forests. Forest Ecology and Mangement, 110:293-314, 1998. 
D. Stoyan and H. Stoyan. On one of Matérns hard-core point process models. Math. Nachr., 122:205-214, 1985.

M. Månsson and M. Rudemo. Random patterns of nonoverlapping convex grains. Adv. appl. Prob., 34:718-738, 2002.

D. J. Daley and D. Vere-Jones. An Introduction to the Theory of Point Processes, Vol. II. Springer, New York, 2008.

D. J. Daley and D. Vere-Jones. An Introduction to the Theory of Point Processes. Springer, New York, 1988.

D. J. Strauss. A model for clustering. Biometrika, 62:467-475, 1975.

J. Møller and R. P. Waagepetersen. Statistical Inference and Simulation for Spatial Point Processes. Chapman \& Hall/CRC, Boca Raton, 2003.

V. L. Gavrikov and D. Stoyan. The use of marked point processes in ecological and environmental forest studies. Environ. Ecolog. Statist., 2:331-344, 1995.

J. Andersson, O. Häggström, and M. Månsson. The volume fraction of a nonoverlapping germ-grain model. Electronic Communications in Probability, 11:78-88, 2006.

R. M. Sok, M. A. Knackstedt, A. P. Sheppard, W. V. Pinczewski, W. B. Lindquist, A. Venkatarangan, and L. Paterson. Direct and stochastic generation of network models from tomographic images: effect of topology on two phase flow properties. Trans. Porous Media, 46:345-371, 2002.

A. Tscheschel and D. Stoyan. On the estimation variance for the specific Euler-Poincaré characteristic of random networks. J. Microsc., 211:80-88, 2003.

P. J. Diggle. Statistical Analysis of Spatial Point Patterns. Arnold, London, 2003.

L. Heinrich. Minimum contrast estimates for parameters of spatial ergodic point processes. In Transactions of the 11th Prague Conference on Random Processes, Information Theory and Statistical Decision Functions, pages 479-492. Academic Publishing House, 1992.

D. Stoyan and H. Stoyan. Estimating pair correlation functions of planar cluster processes. Biometrical J., 38:259-271, 1996. 